\title{
Aldevron Expands to Facilitate Emerging Therapies
}

\section{Introduction}

More than 20 years ago in a small North Dakota State University laboratory, Michael Chambers and John Ballantyne founded Fargo ND-based Aldevron to manufacture DNA plasmids for research applications. Over the next two decades, the company grew steadily, acquiring Freiberg-based Genovac in 2004 for antibody development, and opening a site in Madison, Wisconsin, for recombinant protein design and development in 2009.

Today, Aldevron is the world's leading manufacturer of plasmid DNA, and serves the biotechnology industry with custom production of nucleic acids, proteins, and antibodies.

"Our mission has remained unchanged in 21 years: to help our clients improve lives through innovative partnerships and scientific excellence," says Michael Chambers, CEO of Aldevron. "Our Fargo campus has been, and will continue to be, designed to serve their current and future breakthroughs."

\section{From Research to GMP-Grade Reagents}

Aldevron plays a vital role in the value chain of therapeutic R\&D. It supplies three product grades, research, GMP-Source $^{\mathrm{TM}}$ and GMP. GMP-Source is unique and retains many of the hallmarks of a full GMP batch. Manufacturing occurs in controlled, segregated, non ISO-classified manufacturing suites; QA reviews critical parameters and processes for documentation and complete traceability.

The same CAPA (corrective action/preventative action) system is used for GMP Source and GMP-grade production, and qualified vendors provide the raw materials. Whereas GMP manufacturing takes place in controlled segregated, ISO-classified manufacturing suites with

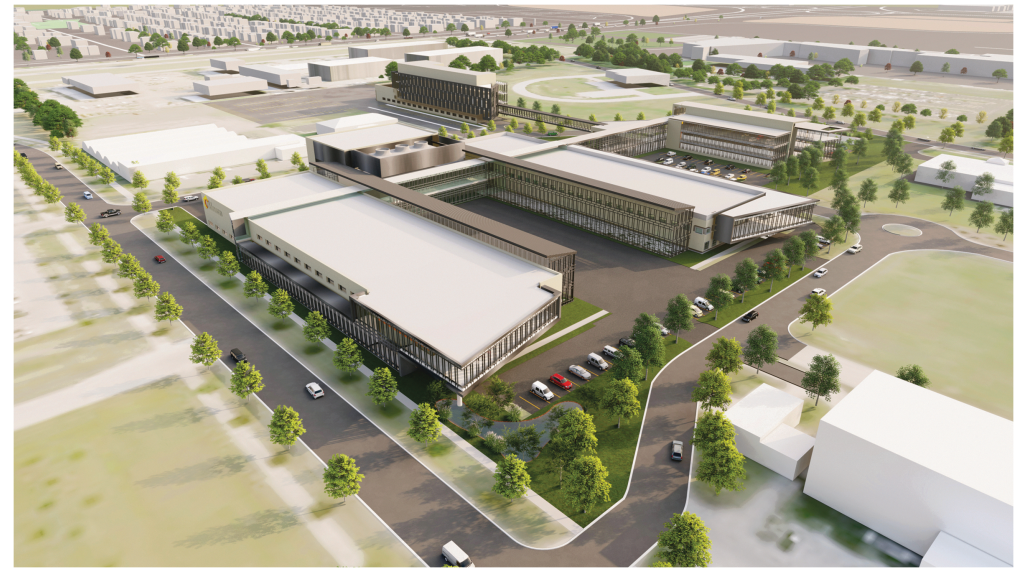

QA review of all documentation, with full change control and equipment validation.

\section{The Basis for Breakthroughs}

Supplying the best reagents to advance science is part of Aldevron's mission. Under a strategic relationship with Integrated DNA Technologies (IDT), Aldevron offers a high-fidelity Cas9 variant, marketed under the trade name, Spy $\mathrm{Fi}^{\mathrm{TM}}$ Cas9 Nuclease, as an off-theshelf product at research, GMP-Source and GMP grades for discovery, clinical and commercial applications. Other offerings include wildtype (WT) SpCas9 Nuclease, AsCpf1 Nuclease and custom manufacturing of other CRISPR-associated nucleases, including dCas9 (dead Cas9), fusions and nickases.

To meet increasing demand for plasmid DNA to produce viral vectors, both for commercial products as well as for the expanding number of development programs, Aldevron initiated standardization and largescale production of helper plasmids. These plasmids are the same regardless of the specific viral vector produced and significantly reduce timelines, costs, and risks. Large-scale batches of the rAAV helper plasmid, pALD-X80, are available for immediate delivery at research and GMP-Source quality grades. Large-scale batches of lentiviral plasmids, pALD-Rev, pALD-VSV$G$ and pALD-GagPol are also available.

\section{Rapid Industry Growth Drives Expansion}

In September 2018, Aldevron opened a new 70,000 square-foot manufacturing facility in Fargo, the largest worldwide for plasmid DNA production, to ramp up capacity and to add capabilities for CRISPR nucleases and enzymatically-synthesized mRNA, a new product line.

Built from the ground up to meet the company's specific needs, the facility houses state-of-the-art equipment, HVAC systems and superior clean rooms. Infrastructure includes a $300 \mathrm{~L}$ single-use fermentation system that increases culturing capacity tenfold and ramped-up downstream processing equipment to yield larger lot sizes.

In early 2019, the Freiberg facility added approximately 4,300 square feet of laboratory and production space. This expansion provided room for personnel growth and equipment for increased cell culture, fusion, production and sequencing capacity, while 


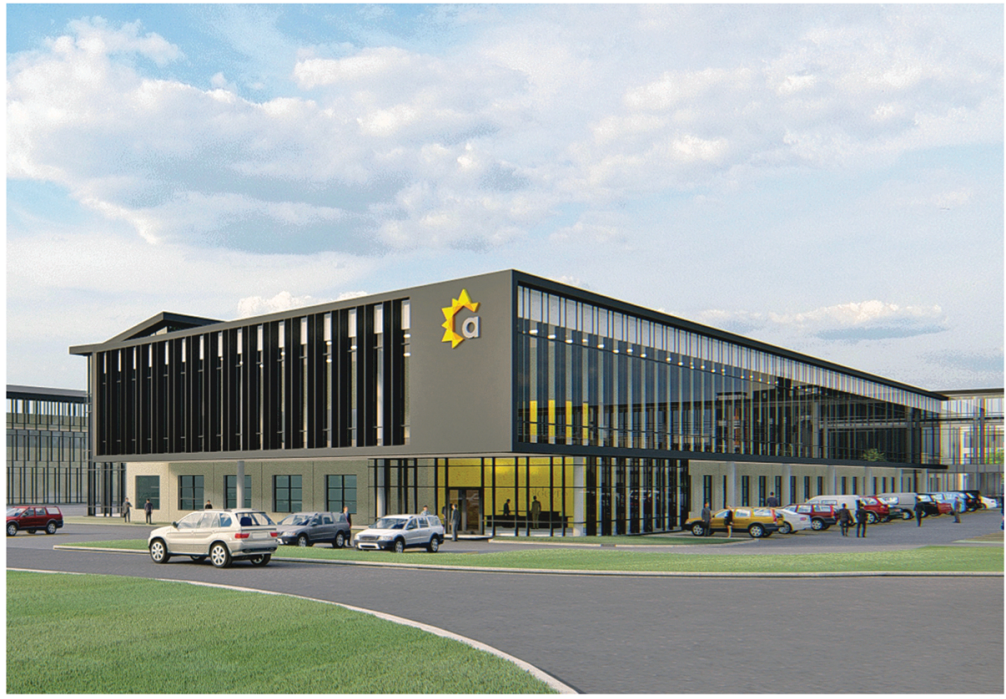

also reserving space for new services to be launched later on.

Aldevron also announced that $1000 \mathrm{~L}$ fermentation capacity will come on-line at the end of 2019 in Madison. The new fermentation capacity will support gene and cell therapy studies that require large amounts of plasmid DNA, recombinant proteins, and/or gene-editing enzymes. This facility expansion takes advantage of an existing \$8-million purposebuilt, bio-manufacturing facility, and adds nearly 10,000 square feet of manufacturing space (with an additional 5,000 square-foot option). A 300L bioreactor for insect and mammalian cell culturing adds capacity to significantly increase manufacturing throughput.

\section{Positioning for the Future}

Aldevron recently announced expansion plans for its 14-acre Fargo campus with phase one construction to begin this summer. Upon completion of the campus, the annual capacity will exceed $\$ 1$ billion of plasmid DNA, RNA, gene-editing enzymes, and other biologics.

The company listened to and engaged with regulatory agencies, industry professionals and, most importantly, clients to design a robust expansion plan that promotes efficiency and scaling, and the next generation of manufacturing optimization.

"The fast-moving gene therapy field requires continuous innovation. The new campus and increased capabilities will give us the ability to innovate faster, and allow us to better inspire, recruit, and retain talent. We will need hundreds of new employees in the coming years. The new buildings will take us to the next level and offer better facilities to help train this workforce, and keep them informed and educated about the latest advances," says CEO Michael Chambers.
According to Chambers, Aldevron strives for continuous improvement. Innovation is central: gene and cell therapy is a new era for medicine. Patients diagnosed with cancer or genetic disease will soon have many more options for treatments and even cures. The R\&D team is growing fast to create new ways to manufacture and deliver these important genetic medicines. The new center will provide a home to collaborate with clients and local universities to better empower the field, and will also help attract the best talent to further accelerate development efforts.

"The campus will be transformative in how it empowers our clients to make genetic medicines for much larger populations. Today, there are a few approved gene therapy products for rare diseases that may have hundreds to a few thousand patients," Chambers says.

"As discoveries advance, soon the field will be producing gene and cell therapies for more "garden variety" conditions, like heart disease, for millions of patients that will require much larger manufacturing platforms. We intend to be equipped to help our clients deliver these new medicines as they advance through the discovery phase and clinical regulatory system."

The first two-story building will increase GMP and GMPSource production capacity up to 10 times its current output. This 189,000 square-foot new building will connect to the existing GMP facility and is expected to be fully operational in early 2021. In total, three new buildings are slated to be built over the next 3-5 years. The additions will enable large-scale production of novel products, such as nanoplasmids and minicircles.

Plans include adding 20,000 square feet of additional QC and product storage space to Aldevron's current 70,000 square-foot GMP and GMP-Source manufacturing building. The next building to be added is an 89,000 square-foot, two-story administration and client visit center, conveniently connected to the manufacturing space via skyway. The final building will be a 96,000 square-foot research and development, technical operations and training center. Once complete, the total square footage will be nearly 0.5 million square feet and has the potential to employ 1,000 people.

Thousands of organizations use Aldevron-produced plasmids, RNA and gene-editing enzymes for projects ranging from basic research to clinical trials to commercial applications. The expansion plan adds the necessary capacity and flexibility to support those discoveries with research, GMP-Source and GMP-grade nucleic acids, proteins and mRNA. Aldevron currently employs more than 350 people across its global network. 